\title{
TNO Recovery, Unlocking the Early Solar System
}

\author{
M. A. Kadooka, K. J. Meech \\ Institute for Astronomy, 2680 Woodlawn Dr., Honolulu, HI 96822 \\ T. Chun
}

Kamehameha Schools, 16-714 Volcano Road, Keaau, HI 96749

\begin{abstract}
.
The recent discoveries of small bodies in the outer solar system in the vicinity of the Kuiper belt are revolutionizing solar system formation concepts by providing critical dynamical and compositional clues to its earliest epochs. However, the rapidly accelerating discovery rate means that follow-up observations for orbit determination cannot keep up. We report here on an innovative test program to assess the potential of a small (2-m class) educational outreach telescope to engage in an efficient recovery program. Recovery observations have been obtained over the course of 22 nights using the University of Hawaii $2.2 \mathrm{~m}$ telescope during 9 observing runs. We targeted over 200 objects and have achieved a $75 \%$ success rate. We obtained time resolved resolved light curve photometry on $2000 \mathrm{OK}_{67}$, and the partial light curve suggests a period $\mathrm{T}>12 \mathrm{hr}$.
\end{abstract}

\section{Introduction}

The prediction of planetary material existing beyond Pluto by Edgeworth (1943) and Kuiper (1951) and advanced CCD technology led to the first discovery of such objects with 1992 QB $_{1}$ (Jewitt \& Luu 1993). These trans-Neptunian objects (TNOs) preserve valuable clues to the dynamical (Holman \& Wisdom 1993; Levison \& Duncan 1997; Malhotra 1995), physical and chemical conditions in the outer solar nebula. Detailed observations are beginning to reveal remarkable physical diversity among these objects. However, the physical study of the objects and the dynamical studies require well determined orbits.

The Deep Ecliptic Survey (DES) was begun in 1998 with a team of observers from Lowell Observatory, the Massachusetts Institute of Technology, the University of California at Berkeley, and more recently the University of Hawaii (UH). The goal of the program is to map out orbital distributions as a function of object class (resonant, non-resonant, and scattered), and to establish accurate orbits so that larger ground-based and space-based telescopes can perform physical studies of these objects. Using large CCD mosaic cameras on the 4-m telescopes at Kitt Peak in Arizona, and at the Cerro Tololo Interamerican Observatory in Chile, the DES is able to image as much sky in a single exposure as the discovery surveys imaged in 5 years (Millis et al. 2002a). As of Sep. 2003, 299 TNOs and 
Centaurs have been discovered with this program (35\% of the total 854 known objects; Millis et al. 2002b; http://www.lowell.edu/Research/DES/).

Unfortunately, discoveries outpace the ability to obtain follow up observations, and many objects are being lost, suggesting the need for dedicated programs. At the same time that the urgent need for dedicated recovery programs was becoming apparent, a new educational telescope, the Faulkes telescope, was being built on the summit of Haleakala on Maui. Because of its large aperture $(2 \mathrm{~m})$, the possibility that students and teachers could become involved in supporting Kuiper belt research was realized. As a pilot test for the new telescope, we developed a recovery program for the UH 2.2-m telescope on Mauna Kea, thus simultaneously assessing the feasibility of of TNO work for high school and college research. As part of the National Science Foundation Teacher Enhancement program, the University of Hawaii's Institute for Astronomy (IfA) hosted 2 teachers (summer 2001) to work on this project.

Complete in late 2003, the aim of the collaboration between the IfA and the Faulkes Telescope Corporation, is to have a dedicated 2- $m$ research-grade telescope for Hawaiian and United Kingdom educational outreach and student research. The facility will be operated remotely from control centers in the UK or Hawaii. The IfA is currently developing an outreach program to train teachers in the fundamental concepts needed to utilize this facility and mentor students, and to select appropriate queue-scheduled research programs. The design of the TNO program is to obtain the necessary orbit refinement astrometry on newly discovered objects while simultaneously identifying interesting candidates for follow up physical studies (e.g. colors, sizes, rotation rates). We report here on the recovery runs, the statistics of the recovered objects and detailed physical observations of one TNO, $2000 \mathrm{OK}_{67}$.

\section{Observational Strategy}

The TNO selection process used in our pilot program was based upon several criteria: brightness, orbital uncertainty, and detector field of view (FOV). TNOs brighter than visual mag 24 were selected from a list of those in need of astrometric measurements to prevent their being lost or to improve their orbits (www.lowell.edu/users/buie/kbo/kbostatus3.html). We selected objects with errors small enough (a few to $\sim 100$ arcsec) to ensure with $3 \sigma$ uncertainty that the TNO would be within the FOV of the detector $(7 \operatorname{arcmin} \times 7$ arcmin). Our strategy was to observe each object twice on one night (for ease in finding the object by blinking the frames), and once on a second night (to verify identification).

\section{Data Acquisition}

Images were taken during 22 nights with two detectors on the UH2.2m telescope (Table 1). The Tek2K detector had a plate scale of 0.219 arsec, a gain of 1.74 $\mathrm{e}^{-} /$ADU (Analog-Digital Units), and a readnoise of $6 \mathrm{e}^{-}$and the Lincoln Labs $2 \times 4 \mathrm{k}$ detector had a plate scale of $0.275 \mathrm{arsec} / \mathrm{pix}$ binned $2 \times 2$, a gain of 1.0 $\mathrm{e}^{-} / \mathrm{ADU}$, and a readnoise of $1.4 e^{-}$. Our filters were based on the Kron-Cousins photometric system $\left(V: \lambda_{o}=5450 \AA, \Delta \lambda=836 \AA ; R: \lambda_{o}=6460 \AA, \Delta \lambda=1245 \AA\right)$. 
All images were guided at sidereal rates with exposure times between $600-900$ sec.

Table 1. TNO Recovery Program

\begin{tabular}{lcccccc}
\hline UT Date & Obs $^{\ddagger}$ & Cond $^{\dagger}$ & Det $^{\varsigma}$ & \#Targ $^{\S}$ & \#Obs $^{\S}$ & \#TNO $^{\S}$ \\
\hline 2001 Feb 25-26 & B & 1,2 & Tek & 18 & 30 & \\
2001 Mar 30-31 & M,R & 1,2 & Tek & 18 & 53 & \\
2001 Apr 26-27 & P & 2,1 & Tek & 16 & 19 & \\
2001 May 19-20 & P & 3,1 & Tek & 12 & 39 & 17 \\
2001 Jul 24-26 & M,F,K,C & $1,1,2$ & Tek & 12 & 47 & \\
2002 Feb 3-7 & M,B,P & $1,1,2,1,1$ & LL & 47 & 90 & 36 \\
2002 Mar 8-9 & P,A & 1,2 & LL & 29 & 26 & 13 \\
2002 Apr 10-11 & B & 2,1 & LL & 32 & 42 & 21 \\
2002 May 8-9 & P,A & 3,3 & LL & 17 & 16 & 8 \\
\hline
\end{tabular}

Note: ${ }^{\ddagger}$ Observers: $B=J$. Bauer, $M=K$. Meech, $R=C$. Ruggles, $P=J$. Pittichová, $\mathrm{F}=\mathrm{Y}$. Fernández, $\mathrm{K}=\mathrm{M}$. Kadooka, $\mathrm{C}=\mathrm{T}$. Chun, $\mathrm{A}=\mathrm{S}$. Ash; ${ }^{\dagger}$ Observing conditions: Phot $=1$, Cirrus $=2$, Cloud $=3 ;{ }^{\zeta}$ Detector; ${ }^{\S} \#$. of attempted targets, actual observations, and observed targets.

\section{Data Reduction}

Images were reduced in the standard manner using the biases and twilight sky flats using the Image Reduction and Analysis Facility (IRAF). Cosmic rays were removed and a bad pixel mask was used to correct for the few bad pixels on the frames. Images were blinked to search for the moving TNOs. The rates of moving objects were calculated and compared to the rates of TNOs as given by the JPL ephemeris to identify each TNO. Aperture photometry was performed on $2000 \mathrm{OK}_{67}$ using the digiphot apphot package in IRAF. This routine locates the image centroid within the user-defined circular aperture. The sky background was obtained from an annulus which lies just beyond the photometry aperture. The errors quoted for the final reduced photometry include both the statistical photon noise of the measurements and the external errors associated with the transformation to the standard system. Because the objects are faint, and the sky noise dominates the errors, an aperture radius of $2.5 \operatorname{arcsec}$ was used.

Standard stars from Landolt (1992) were observed on each photometric night, and the nights were calibrated individually using stars of a wide range of colors, with extinction determined over a range of airmasses similar to or greater than the observed objects. Cirrus was present on the night of 2001 July 26 (the night with most of our $2000 \mathrm{OK}_{67}$ photometry), and no standard star data were obtained. We performed photometry on 10 field stars in each image, and then used differential photometry to correct for the variable extinction. These data are presented in Table 2. We used the brightest values of the field stars during a period of apparent stability to estimate the true brightnesses, however, these data need photometric recalibration. This method of differential photometry works well for removing non-photometric variations up to $\approx 0.5$ 
Table 2. $\quad 2000 \mathrm{OK}_{67}$ Magnitudes, July 26, 2001

\begin{tabular}{cccc}
\hline MidUT & $\chi$ & Exp & Mag $\pm \sigma$ \\
\hline 08.4156 & 2.850 & 900 & $22.503 \pm 0.091$ \\
09.0869 & 2.059 & 900 & $22.634 \pm 0.123$ \\
09.4306 & 1.824 & 900 & $22.662 \pm 0.076$ \\
09.7269 & 1.672 & 900 & $22.714 \pm 0.075$ \\
10.0161 & 1.555 & 900 & $22.729 \pm 0.095$ \\
10.3042 & 1.462 & 900 & $22.803 \pm 0.087$ \\
10.5931 & 1.388 & 900 & $22.706 \pm 0.071$ \\
11.0769 & 1.297 & 900 & $22.673 \pm 0.069$ \\
11.3436 & 1.256 & 600 & $22.700 \pm 0.095$ \\
13.7936 & 1.269 & 600 & $22.721 \pm 0.163$ \\
\hline
\end{tabular}

mag of extinction. The differential correction and the corrected light curve for $2000 \mathrm{OK}_{67}$ on 2001 July 26 are shown in Figure 1.
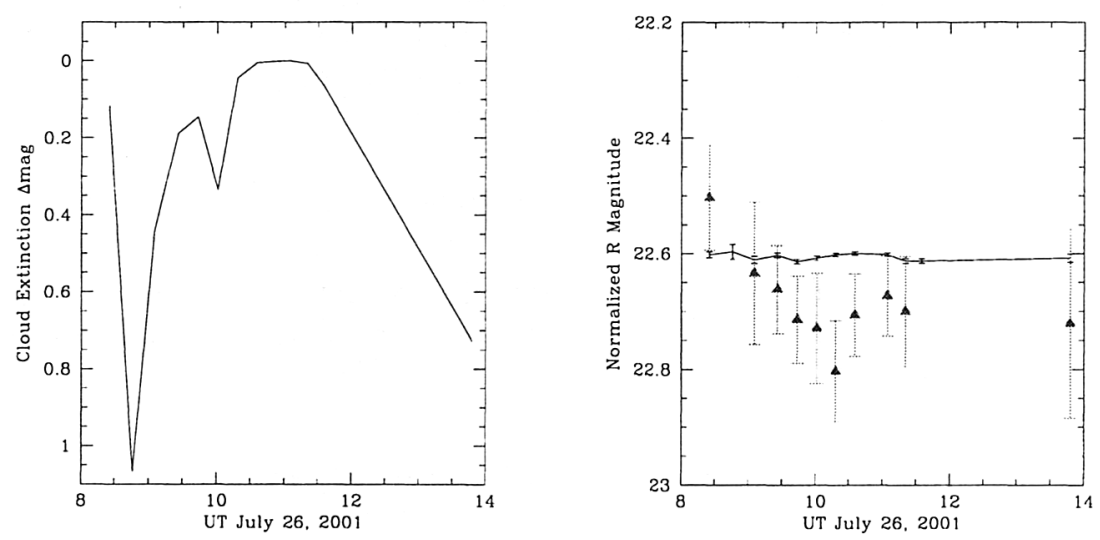

Figure 1. [a]Variable extinction on 2001, July 26; [b]The $2000 \mathrm{OK}_{67}$ light curve compared to the normalized average of 10 field stars.

\subsection{Astrometry}

IDL software written by M. Buie, Lowell Observatory, was used to perform the astrometric reduction of the frames. The software is a cubic astrometric solution which includes 10 terms and is based on the USNO-A2.0 catalog. The typical errors were around 0.1 arcsec. On many of the runs we made recoveries, and on others discovery arc extensions which will help with future recoveries, and for several objects we saved them from being lost. The statistics of the recovery observations are presented in Table 1 . 


\section{Discussion}

There is an insufficient time baseline to find the period for $2000 \mathrm{OK}_{67}$ from the existing data, however, it is clear that there is a time variable signature, which is probably caused by rotation. A possible minimum in the light curve was seen during the 3 hour observing interval, but we don't know if the light curve reached a maximum. This suggests the full change in brightness occurs on a timescale $\geq 3 \mathrm{hr}$. If this is due to an irregular shape for the object, then this would correspond to a period $\mathrm{T}>12 \mathrm{hr}$. The only way to distinguish a light curve due to shape from one due to albedo or compositional features on the surface would be to search for time resolved color differences.

Using $\mathrm{m}_{R}=22.6$ as the average brightness for $2000 \mathrm{OK}_{67}$, we can estimate the radius for the $2000 \mathrm{OK}_{67}$ from:

$$
p_{R} R_{N}{ }^{2}=\frac{2.24 \times 10^{22} r^{2} \Delta^{2} 10^{0.4\left(m_{\odot}-m\right)}}{10^{-\beta \alpha}}
$$

where $r=40.788 \mathrm{AU}, \Delta=39.896 \mathrm{AU}$, and the phase angle $\alpha=0.70$. The radius, $R_{N}=143.8 \mathrm{~km}$ for a phase function $\beta=0.035 \mathrm{mag} \mathrm{deg}^{-1}$ (Meech \& Jewitt 1987) and assuming a geometric albedo, $p_{R}=0.04$, compares well to the value that Delsanti et al. (2001) obtained of $159.3 \mathrm{~km}$ for their brightness estimate of 22.24. The observed brightness change, $\Delta \mathrm{m}>0.3 \mathrm{mag}$, translates to a lower limit of the nucleus axial ratio a:c $>1.3: 1$ from:

$$
a: c=10^{+0.4 \Delta m} \text {. }
$$

Although an object as large as $2000 \mathrm{OK}_{67}$ might be expected to be more nearly spherical, by calculating the central pressure, $P_{\text {cent }}$, assuming a constant density, $\rho$ throughout:

$$
P_{\text {cent }}=\frac{2}{3} \pi G \rho^{2} r^{2}=1.4 \times 10^{-10} \rho^{2} r^{2}
$$

we get $P_{\text {cent }}=7 \times 10^{5}-3 \times 10^{6}$ for a plausible range of comet densities which is smaller than or comparable to the strength of chondritic material $\left(S=6 \times 10^{6}\right.$ $\left.4 \times 10^{8} \mathrm{Nm}^{-2}\right)$. This is somewhat stronger than cometary material strengths $\left(S=10^{3}-10^{5} \mathrm{Nm}^{-2}\right)$, however. The smaller TNOs are likely to be collisional fragments, and the interiors may be strong enough to withstand gravitational pressure, so that objects this size need not necessarily be spherical.

\subsection{Comparison to Other TNO Light Curves}

Since the focus has been on the discovery and recovery of TNOs, and because the objects are faint, there have been limited physical observations of these objects, including only a small number of published light curves and rotation periods. Table 3 summarizes the known TNO rotation periods. The partial signature of the rotation period found for $2000 \mathrm{OK}_{67}$ is entirely consistent with the rotation rates found for other TNOs to date. 
Table 3. Optical Light Curves of Centaurs and TNOs

\begin{tabular}{lcccl}
\hline Object & $\mathbf{P}[\mathbf{h r s}]^{\dagger}$ & $\Delta \mathbf{m}^{\ddagger}$ & $\mathbf{a : c}^{\zeta}$ & Refs \\
\hline Chiron & 5.9 & 0.03 & 1.02 & Bus et al., 1988 \\
Pholus & 9.98 & 0.1 & 1.1 & Buie \& Bus, 1992 \\
Asbolus & 8.9 & 0.3 & 1.3 & Brown, \& Luu, 1997 \\
$1998 \mathrm{BU}_{48}$ & 9.8 & 0.68 & 1.9 & Sheppard \& Jewitt, 2002 \\
$1999 \mathrm{UG}_{5}$ & 13.41 & 0.10 & 1.1 & Gutiérrez et al., 2001 \\
$2001 \mathrm{PT}_{13}$ & 8.4 & 0.15 & 1.1 & Farnham, 2001 \\
$1994 \mathrm{~TB}$ & 3 & 0.15 & 1.1 & Romanishin \& Tegler, 1999 \\
$1994 \mathrm{VK}_{8}$ & 4.7 & 0.21 & 1.2 & Romanishin \& Tegler, 1999 \\
$1995 \mathrm{QY}_{9}$ & 7.3 & 0.60 & 1.7 & Sheppard \& Jewitt, 2002 \\
Varuna & 6.34 & 0.42 & 1.5 & Sheppard \& Jewitt, 2002 \\
$1999 \mathrm{KR}_{16}$ & 11.8 & 0.18 & 1.2 & Sheppard \& Jewitt, 2002 \\
$2000 \mathrm{GN}_{171}$ & 8.33 & 0.61 & 1.7 & Sheppard \& Jewitt, 2002 \\
$1996 \mathrm{TO}_{66}$ & 6.25 & 0.16 & 1.2 & Hainaut et al., 2000 \\
1998 SM $_{165}$ & 7.1 & 0.45 & 1.5 & Sheppard \& Jewitt, 2002 \\
\hline
\end{tabular}

Notes: ${ }^{\dagger}$ Rotation period in hrs (the double-peaked period is reported here); ${ }^{\ddagger}$ Light curve range; ${ }^{\zeta}$ minimum axial ratio of TNO.

\section{Conclusions}

The year-long TNO recovery program presented here has been extremely productive and has yielded the following results:

- This pilot program has the capability of a small 2-m class telescope, such as the Faulkes telescope, to efficiently achieve astrometric measurements sufficient for orbital refinement for relatively faint TNOs;

- Approximately $75 \%$ of the objects attempted were found, for a total of 150 objects over 22 nights of observation;

- $2000 \mathrm{OK}_{67}$ exhibits brightness variations consistent with either a light curve caused by surface albedo features with a period $>6 \mathrm{hrs}$, or a period $>12 \mathrm{hrs}$ if caused by shape. This is consistent with other reported TNO rotation periods;

- The light curve range, $\Delta \mathrm{m}>0.3 \mathrm{mag}$, implying an axial ratio a:c $>1.3$, if caused by shape;

- We estimate a minimum radius of $143.8 \mathrm{~km}$ for $2000 \mathrm{OK}_{67}$.

\section{Acknowledgments}

We wish to thank J. Bauer, J. Pittichová, and Y. Fernández for their assistance with the observations and data reduction. Image processing was done using the IRAF program distributed by the National Optical Astronomy Observatories, 
which is operated by the Association of Universities for Research in Astronomy, Inc. (AURA) under cooperative agreement with the National Science Foundation. This research was supported in part by a grant from the National Science Foundation Research Experiences for Teachers program (\#AST-9987896), and a grant from NASA (NAG5-4495). M. Kadooka acknowledges the receipt of the Australian-American Fulbright Symposium 2002 for a Travel grant subsidy to present this work at the 2002 Bioastronomy meeting.

\section{References}

Brown, W. R. \& Luu, J. X. 1997, Icarus, 126, 218

Buie, M. W. \& Bus, S. J. 1992, Icarus, 100, 288

Bus, S. J., Bowell, E., \& French, L. M. 1988, IAUC, 4684, 2

Edgeworth, K. E. 1943, J. Br. Astron. Assoc., 53, 181

Delsanti, A. C., Boehnhardt, H., Barrera, L., Meech, K. J., Sekiguchi, T., \& Hainaut, O. R. 2001, A\&A, 380, 347

Farnham, T. L. 2001, BAAS, 33, 31.04

Gutiérrez, P. J., Ortiz, J. L., Alexandrino, E., Roos-Serote, M., \& Doressoundiram A., 2001, A\&A, 371, L1

Hainaut, O. R., Delahodde, C. E., Boehnhardt, H., Dotto, E., Barucci K. J., Meech, M. A., Bauer, J. M., West, R. M., \& Doressoundiram, A. 2000, A\&A, 356, 1076

Holman, M. J. \& Wisdom, J. 1993, AJ, 105, 1987

Jewitt, D. C. \& Luu, J. X. 1993, Nature, 362, 6422, 730

Kuiper, G. P. (1951), in Astrophysics, ed. J. A. Hynek, New York, McGraw-Hill, 357

Landolt, A. U. 1992, AJ, 104, 340

Levison, H. F. \& Duncan, M. J. 1997, Icarus, 127, 13

Malhotra, R. 1995, AJ, 110, 420

Meech, K. J. \& Jewitt, D. C. 1987, Astron. Astrophys., 187, 585

Millis, R. L., Buie, M. W., Wasserman, L. H., Elliot J. L., Kern, S. D., \& Wagner, R. M. 2002a, AJ, 123, 2083

Millis, R. L., Buie, M. W., Wasserman, L. H., Elliot, J. L., Kern, S. D., Clancy, K., Wagner, R. M., Chiang, E. I., Jordan, A., Trilling, D. E., \& Meech, K. J. 2002b, BAAS, 34, \#9.03.

Rominishin, W., \& Tegler, S. C. 1999, Nature, 398, 129

Sheppard, S. S., \& Jewitt, D. C. 2002, AJ, 124, 1757 